\title{
Nonlinear Band Structure in Bose Einstein Condensates: The Nonlinear Schrödinger Equation with a Kronig-Penney Potential
}

\author{
B. T. Seaman, L. D. Carr and M. J. Holland \\ JILA, National Institute of Standards and Technology and Department of Physics, \\ University of Colorado, Boulder, CO 80309-0440
}

(Dated: June 22, 2018)

\begin{abstract}
All Bloch states of the mean field of a Bose-Einstein condensate in the presence of a one dimensional lattice of impurities are presented in closed analytic form. The band structure is investigated by analyzing the stationary states of the nonlinear Schrödinger, or Gross-Pitaevskii, equation for both repulsive and attractive condensates. The appearance of swallowtails in the bands is examined and interpreted in terms of the condensates superfluid properties. The nonlinear stability properties of the Bloch states are described and the stable regions of the bands and swallowtails are mapped out. We find that the Kronig-Penney potential has the same properties as a sinusoidal potential; Bose-Einstein condensates are trapped in sinusoidal optical lattices. The Kronig-Penney potential has the advantage of being analytically tractable, unlike the sinusoidal potential, and, therefore, serves as a good model for experimental phenomena.

PACS numbers: 03.75.Hh,05.03.Jp,03.65.Ge
\end{abstract}

\section{INTRODUCTION}

Periodic potentials are ubiquitous in physics, appearing in electron transport in metals [1], Josephson junction arrays 2], nonlinear photonic crystals and waveguide arrays [3], and Bose-Einstein condensates (BEC's) [4]. With the realization of BEC's of alkali atoms in a sinusoidal optical lattice, there has been an explosion in studies of BEC's in periodic potentials, both experimentally and theoretically $[5,6,6,6,8,010]$. BEC's in periodic potentials have been used to study phase coherence of atom lasers [11, 12] and matter-wave diffraction [13]. Therefore, in the context of the BEC, the study of periodic potentials provides an excellent connection between condensed matter physics and atomic physics. In contrast to other physical contexts, the lattice geometry and strength, as well as the interatomic interactions [14, 15], are all tunable parameters for the BEC. We show that the mean field Bloch states of a BEC in a Kronig-Penney potential, i.e., a lattice of delta functions, exhibits the same band structure and stability properties as the experimental case of a sinusoidal potential. Unlike in the case of the sinusoidal potential, Bloch state solutions to the KronigPenney potential can be described by straightforward analytic expressions. The Kronig-Penney potential has distinct advantages as a model of BEC's trapped in periodic potentials.

Specifically, we consider the steady state response of the mean field of a BEC to a Kronig-Penney potential using the Bloch ansatz. The mean field is modeled by the nonlinear Schrödinger equation (NLS), which appears in numerous areas of physics; in the context of the BEC, it is often called the Gross-Pitaevskii equation 16, 17]. We have previously obtained the full set of stationary solutions for a single delta function analytically [18, 19, 20, 21]. Using these results, we are able to rigorously describe the band structure. We find that above a critical nonlinearity, swallowtails, or loops, form in the bands 22, 23. These swallowtails are related to superfluid properties of the BEC 24], as we shall explain. Stability properties are studied numerically by time evolution of perturbed stationary state solutions to the NLS. It is found that stable, as well as unstable, regimes exist for both repulsive and attractive BEC's. We also elucidate the linear Schrödinger and discrete nonlinear Schrödinger limits of our model. The latter limit is related to the superfluid phase of the BoseHubbard model [25].

Experimentally, in order to create a BEC in a lattice, alkali atoms are first cooled to a quantum degenerate regime by laser cooling and evaporation in harmonic electromagnetic traps 26, 27]. A sinusoidal optical lattice is then created by the interference of two counterpropagating laser beams, which creates an effective sinusoidal potential proportional to the intensity of the beams [10]. The potential is due to the AC Stark shift induced by the dipole interaction with the electromagnetic field on the atoms' center of mass 28]. For large detuning of the optical field from the atomic transition, dissipative processes, such as spontaneous emission, can be minimized and the potential becomes conservative. Nonzero quasimomentum can be examined by slightly detuning the two lasers by a frequency $\delta \nu[\underline{6}]$. The resulting interference pattern is then a traveling wave moving at the velocity, $v=(\lambda / 2) \delta \nu$, where $\lambda$ is the wavelength of the first beam. This produces a system with quasimomentum $q=m v / \hbar$, where $m$ is the atomic mass. After a given evolution time, the traps are switched off. The BEC is allowed to expand and the density is then imaged.

The sinusoidal optical lattice potential is composed of a single Fourier component. If more counter-propagating laser beams of different frequencies are added, more Fourier components are introduced, and the potential becomes a lattice of well separated peaks. In the limit that the width of the these peaks becomes much smaller than the healing length of the BEC, the potential effectively 
becomes a Kronig-Penney lattice.

We proceed to highlight a few of the many experiments on BEC's trapped in lattice potentials. In a work by Anderson and Kasevich [1] , the tunneling of a BEC between sites of an optical lattice aligned with the gravitational field was examined. This produced the matter wave analog of the AC Josephson effect, as well as a new kind of atom laser. Greiner et al. 10] observed a quantum phase transition in a 3D lattice from a superfluid to a Mott-insulator. BEC's trapped in lattices have been proposed as one possible realization of a quantum computer and many studies have been performed in this direction, see for example 29] and references therein. An optical lattice was used to renormalize the effective mass to create a Tonks-Girardeau gas [30, 31, 32], which describes a truly one dimensional Bose gas. With regards to band structure, Fallani et al. [6], investigated the dynamic instability of a BEC in a lattice for various quasimomenta. By determining the rate of loss due to heating at a given quasimomentum, they were able to compare their data with theoretically predicted instability growth rates. We emphasize that our study treats the quasi one dimensional regime in which the BEC is always in a superfluid state and can be modeled by the NLS with a periodic potential, as was the case in the experiment of Fallani et al.

The case of a BEC trapped in a sinusoidal potential has been studied theoretically in great detail by a number of researchers 22, 23, 24, 25, 33, 34, 35, 36, 37, 38, 39, 40, 41, 42, 43, 44, 45. Analytic results were restricted to certain special cases 40, 43, 44, 45]; the full band structure was obtained numerically, as for example, by expansion of the wavefunction in Fourier modes [22]. Many of these authors considered stability properties of stationary states. We will compare our results to those of Machholm et al. 22]. A generalized periodic potential in the form of a Jacobian elliptic function has been studied with mathematical rigor 43, 44, 45]. A subset of the solutions to the Kronig-Penney potential has been found 46, 47, 48, 49, 50. Recently, for example, Li and Smerzi 46], investigated generalized Bloch states for constant phase and zero current.

The article is organized as follows. In Sec. III the Bloch wave solutions to the stationary NLS with a KronigPenney potential are presented. The quasimomentumenergy bands are detailed for repulsive and attractive condensates in both the weakly and strongly interacting regimes in Sec. [II] and Sec. IV] In Sec. $\nabla$ the changes in the density profile of the condensate is examined as the quasimomentum changes. In Sec. VI] various limiting cases of the NLSE are examined. The stable and unstable regimes of the bands are studied in detail for both repulsive and attractive condensates in Sec VII. Finally, concluding remarks are made in Sec. VIII]

\section{THE NONLINEAR SCHRÖDINGER EQUATION AND BLOCH WAVES}

In this paper, we consider the mean-field model of a quasi-1D BEC in the presence of a Kronig-Penney potential,

$$
V(x)=V_{0} \sum_{j=-\infty}^{+\infty} \delta(x-j d) .
$$

where $d$ is the lattice spacing and $V_{0}$ is the strength of the potential. When the transverse dimensions of the BEC are on the order of its healing length, and its longitudinal dimension is much longer than its transverse ones, the 1D NLS [51] which describes the stationary states of the mean field of a BEC is given by,

$$
-\frac{1}{2} \Psi_{x x}+g|\Psi|^{2} \Psi+V(x) \Psi=\mu \Psi .
$$

Note that harmonic oscillator confinement in the transverse directions with frequency $\omega$ has been assumed for atoms of mass $m$ and $\mu$ is the eigenvalue, $g$ characterizes the short range pairwise interaction, and $V(x)$ is an external potential [52. In this paper, a weakly interacting system is defined by, $g n / V_{0} \leq 1$, and a strongly interacting system is defined by, $g n / V_{0} \gg 1$. In the case where the harmonic oscillator length approaches the $s$ wave scattering length, $a_{s}$, the 1D NLS no longer models the system and a one-dimensional field theory with the appropriate effective coupling constant must be considered instead [52]. Since $a_{s}$ is on the scale of hundreds of angstroms for typical BEC's, this regime is not relevant to the present study.

In Eq. (2), the length is scaled according to the lattice spacing, $d$, and energy has been rescaled by $\pi^{2} /\left(2 E_{0}\right)$, where

$$
E_{0} \equiv \frac{\hbar^{2} \pi^{2}}{2 m d^{2}},
$$

is the kinetic energy of a particle with wave vector equal to that at the boundary of the first Brillouin zone. The variables in Eq. (2) are defined by,

$$
\begin{aligned}
x & =\frac{1}{d} x^{\prime}, \\
\mu & =\frac{\pi^{2}}{2 E_{0}} \mu^{\prime}, \\
g & =\frac{\pi^{2}}{2 E_{0}} g^{\prime}, \\
V(x) & =\frac{\pi^{2}}{2 E_{0}} V^{\prime}\left(x^{\prime} / d\right),
\end{aligned}
$$

where the primed variables contain the physical units of the system. The renormalized 1D coupling is, $g n \equiv$ $2 n a_{s} \omega_{\perp} \hbar m d^{2} / \hbar^{2}$, where $n$ is the average density per lattice site. Both attractive and repulsive atomic interactions, i.e., $g>0$ and $g<0$, shall be considered. The 
wave function or order parameter $\Psi(x, t)$ has the physical meaning of,

$$
\Psi(x, t)=\sqrt{\rho(x, t)} \exp [-i \mu t+i \phi(x, t)],
$$

where $\rho(x, t)$ is the line density and the superfluid velocity is given by $v(x, t)=(\hbar / m) \partial \phi(x, t) / \partial x$.

In addition to the NLS, Eq. (2), the normalization of the wavefunction is given by,

$$
n=\int_{0}^{1} \rho\left(x^{\prime}\right) \mathrm{d} x^{\prime},
$$

where $n$ is the number of atoms per lattice site. The boundary conditions induced by the Kronig-Penney potential causes a discontinuity in the derivative of the wavefunction across each delta function,

$$
\partial_{x} \rho(j+\epsilon)-\partial_{x} \rho(j-\epsilon)=4 V_{0} \rho(0),
$$

where $j$ is an integer and $\epsilon \rightarrow 0$.

A brief review is now given of the general solution to Eq. (2) with no external potential. We have previously presented a proof that this represents the full set of solutions for a constant potential [18]. Therefore, by using this complete set of stationary state solutions to the constant potential case, we can calculate the full set of Bloch solutions for a lattice. With the solutions in the form of Bloch waves, the relationship between the energy per particle and the quasimomentum of the Bloch waves is determined.

The constant potential solutions are the form of the density in each lattice site. The density, $\rho$, and the phase, $\phi$, are,

$$
\begin{aligned}
& \rho(x)=B+\frac{k^{2} b^{2}}{g} \operatorname{sn}^{2}\left(b x+x_{0}, k\right), \\
& \phi(x)=\alpha \int_{0}^{x} \frac{1}{\rho\left(x^{\prime}\right)} \mathrm{d} x^{\prime},
\end{aligned}
$$

where $s n$ is a Jacobi elliptic function [53, 54] and the density offset, $B$, the horizontal scaling, $b$, the translational offset, $x_{0}$, and the elliptic parameter, $k$, are free variables. The Jacobi elliptic functions are generalized periodic functions characterized by an additional parameter, $k \in[0,1]$. In the limit that $k \rightarrow 0$ and $k \rightarrow 1$, the Jacobi elliptic functions become circular and hyperbolic trigonometric functions, respectively. The period of the square of the Jacobi elliptic functions is given by $2 K(k)$, where $K(k)$ is the complete elliptic integral of the first kind [53, 54].

Inserting Eqs. (11) and (12) into Eqs. (8) and (2), with $V(x)=0$, one finds that the eigenvalue, $\mu$, and phase prefactor, $\alpha$, are given by,

$$
\begin{aligned}
\mu & =\frac{1}{2}\left(b^{2}\left(1+k^{2}\right)+3 B g\right), \\
\alpha^{2} & =B\left(k^{2} b^{2} / g+B\right)\left(b^{2}+B g\right) .
\end{aligned}
$$

Note that the fact that $\alpha$ only enters into the equations as $\alpha^{2}$ implies that all nontrivial phase solutions, i.e., those for which $\alpha \neq 0$, are doubly degenerate, as $\pm \alpha$ lead to the same value of the eigenvalue, $\mu$, without otherwise changing the form of the density or phase. The boundary conditions are used to determine the appropriate values of the variables.

After using the methods from the previous paper [18], the solutions are now represented in the usual Bloch form,

$$
\Psi(x)=e^{i q x} f_{q}(x),
$$

where $q$ is the quasimomentum and $f_{q}(x)$ has the same period as the lattice, $f_{q}(x)=f_{q}(x+1)$. By substituting Eq. (15) into Eq. (8), one finds that the density, $\rho$, must also have the same period as the lattice and that the quasimomentum and energy per particle can be determined from the density profile by,

$$
\begin{aligned}
q & =\int_{0}^{1} \frac{\alpha}{\rho\left(x^{\prime}\right)} \mathrm{d} x^{\prime} . \\
\frac{E[\Psi]}{n} & =\frac{1}{n} \int_{0}^{1} \mathrm{~d} x\left(\frac{1}{2}\left|\Psi_{x}\right|^{2}+\frac{g}{2}|\Psi|^{4}+V_{0} \delta(x)|\Psi|^{2}\right)(17)
\end{aligned}
$$

The quasimomentum is simply the phase jump across each lattice site and corresponds to the momentum due to the superfluid velocity of the system. Since nontrivial phase solutions are degenerate for the two phase prefactors, $\pm \alpha$, only half of a Brillouin zone needs to be calculated, i.e. $0 \leq q \leq \pi$. The second half will then be a reflection around $q=0$.

This paper examines the quasimomentum-energy bands and, therefore, reduces the problem to a situation where the density is symmetric around the ends, $x=j$ or $x=j+1$, and the middle, $x=j+0.5$, of the lattice sites, where $j$ is an integer. Due to this symmetry, there are only two possible values for the translational offset, $x_{0}$,

$$
x_{0} \in\left\{-\frac{b}{2}, K(k)-\frac{b}{2}\right\},
$$

where $K(k)$ is the half period of the density. The offset forces the density in the center of each site to be either a minimum or a maximum of the site, depending on the sign of the interaction.

Since it is computationally intensive to include the integral in the quasimomentum equation, Eq. (16), with a root finding algorithm, one of the parameters, $b, k$, or $B$, is varied while the other two are determined from the number equation, Eq. (9), and boundary condition, Eq. (10). The quasimomentum and energy are evaluated from these parameters and can then be plotted parametrically. In the following two sections, we discuss the energy bands from repulsive and attractive interatomic interactions.

\section{REPULSIVE ATOMIC INTERACTIONS}

The structure of the energy bands is strongly dependent on the strength and sign of the atom-atom inter- 


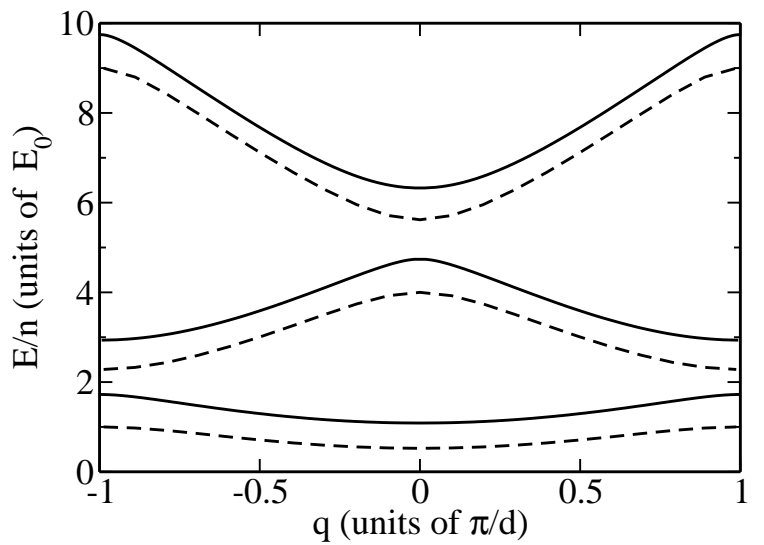

FIG. 1: Energy per particle as a function of the quasimomentum for the first three bands of a weakly repulsive condensate $\left(g n=E_{0}\right)$ in a repulsive lattice $\left(V_{0}=E_{0}\right)$. The noninteracting linear band structure is given by the dashed curves.

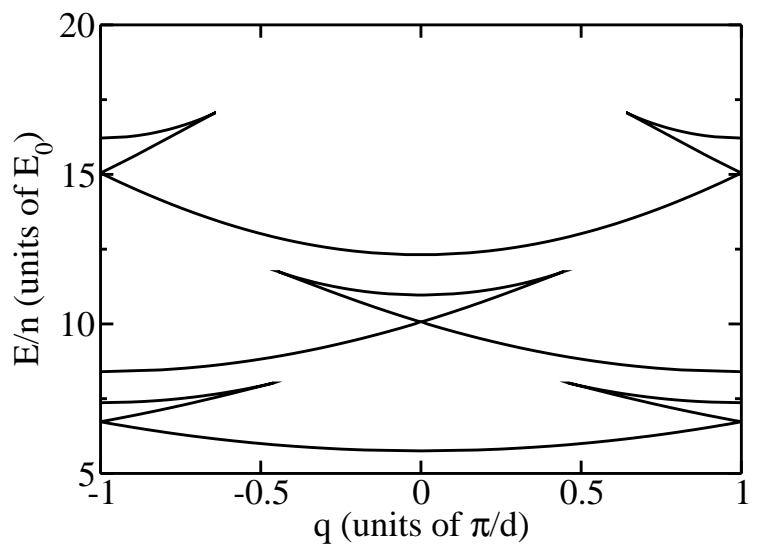

FIG. 2: Energy per particle as a function of the quasimomentum for the first three bands of a strongly repulsive condensate $\left(g n=10 E_{0}\right)$ in a repulsive lattice $\left(V_{0}=E_{0}\right)$.

actions, g. In Fig. प and Fig. 2] the energy bands for specific cases of weak and strong repulsive interactions are presented, $g n=E_{0}$ and $g n=10 E_{0}$, respectively. The condensates are assumed to be in a repulsive lattice, $V_{0}=E_{0}$. Note that the energies are given as unscaled values so that easy comparison with previous literature is possible.

Notice that in Fig. 1 the interaction strength is small and deviations from the linear band structure are small as well. The bands are vertically shifted higher as compared to a linear system due to the repulsive interactions that increase the energy of the system. When the interaction strength is further increased the band structure becomes quite different. Swallowtails 38] appear at the ends of the bands, as in Fig. 22 The width of these swallowtails grows as the interaction strength is increased. Swallowtails are a general feature of a nonlinear system

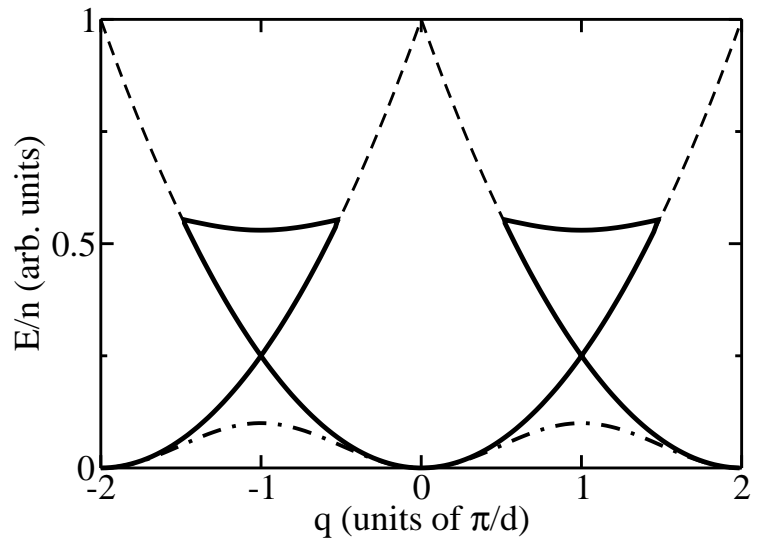

FIG. 3: Energy per particle as a function of the quasimomentum wave number for a noninteracting system with no potential (dashed curve), a periodic potential and a small or no interaction (dot-dashed curve) and a periodic potential with a large interaction (solid curve).

in a periodic potential [42] and appear for both repulsive and attractive interactions.

The presence of these swallowtails is due to the hysteric behavior of the superfluid condensate. A thorough discussion of this topic is given by Mueller 24] (see also references therein). For a completely free, noninteracting system, the energy has a quadratic dependence on the momentum, shown as the dashed parabolic curves in Fig. 3 Since a quasimomentum of $2 \pi$ leaves the system unchanged, the quadratic dependence is repeated centered around integer multiples of $2 \pi$. When a periodic potential is added to the system, bands in the energy are formed, shown as the dot-dash sinusoidal curve in Fig. 3 These bands may be found by solving the linear Schrödinger equation. An interacting condensate, however, is a superfluid and can therefore screen out the periodic potential [41]. The energy band will then appear similar to that of a free particle, shown as a solid swallowtail curve in Fig. 3 until a critical point. Since there is a critical velocity, determined by the condensate sound speed, the energy band must terminate. If this velocity allows the quasimomentum to pass the edge of the Brillouin zone, there are then two separate energy minima. This demands that there be a saddle point separating them and hence the three stationary states. For a Kronig-Penney potential, just as for a sinusoidal potential, when a swallowtail appears there are two spinodal points, where the number of energy extrema changes, at the edges of the swallowtail. These are points where the local minimum and local maximum energy converge to the same energy. As the interaction strength is increased, the width of the swallowtail can increase without limit. Eventually, the swallowtail will become large enough to cross the higher bands. In this case, there is a degeneracy between bands.

For interacting systems in periodic potentials, there is 
a minimum interaction strength for which the swallowtails in the energy bands can exist 24]. This can, in general, be dependent on both the strength of the potential as well as the band that is being discussed. For an optical lattice it was shown that the onset of the swallowtail for the lowest band of a repulsive condensate occurs when the interaction strength and the potential are equal. For higher bands the relationship no longer becomes analytic [22]. For the Kronig-Penney lattice potential, the critical value for the onset of the swallowtails is not dependent on the band under consideration or the sign of the interaction. Numerically, we are able to determine that the onset occurs when $g n=2 V_{0}$. The factor of two is not present for the optical lattice potential. This holds true in all situations except for the lowest band of an attractive condensate, as will be explained in the following section.

The energy bands are slightly different when the condensate is in an attractive potential, i.e. $V_{0}<0$. At the Brillouin zone boundary, the energy gap between bands is proportional to $V_{0}$ for a weakly interacting system. The density is an equally spaced array of solitons. With a quasimomentum of $q=\pi$ in the lowest band, the condensate has one dark soliton [55] per lattice site with nodes at the delta functions. The potential, therefore, does not contribute to the total energy of the system since the delta function potential only depends on the density at that position. The second band with quasimomentum of $q=\pi$, however, has a density that is offset by half a period. There is then a dark soliton in the center of each lattice site. The effect of the potential lowers the energy by $\rho(0) V_{0}$. The second band then can cross the first band for a condensate in an attractive potential. In contrast, the bands are separated by an energy $\rho(0) V_{0}$ for a repulsive potential. As the interaction strength increases, the effects of the potential become less noticeable and the bands are no longer degenerate. Note that repulsive and attractive sinusoidal potentials create the same band structure. The difference in the current system arises since there are two length scales associated with a Kronig-Penney potential, the lattice spacing and delta function width.

\section{ATTRACTIVE ATOMIC INTERACTIONS}

The energy bands for an attractive condensate in a repulsive potential with a small interaction strength have a qualitatively similar form as for a weakly repulsive condensate. Note that the attractive bands are, however, lower in energy than the repulsive bands. The attractive bands are pushed down from the linear case due to the attractive interaction strength. A strongly attractive condensate, however, has several qualitative differences compared to a strongly repulsive condensate.

In Fig. 4 the band structure for a small attractive interaction, $g n=-E_{0}$, in a repulsive potential, $V_{0}=E_{0}$, is illustrated. The band structure for this system is al-

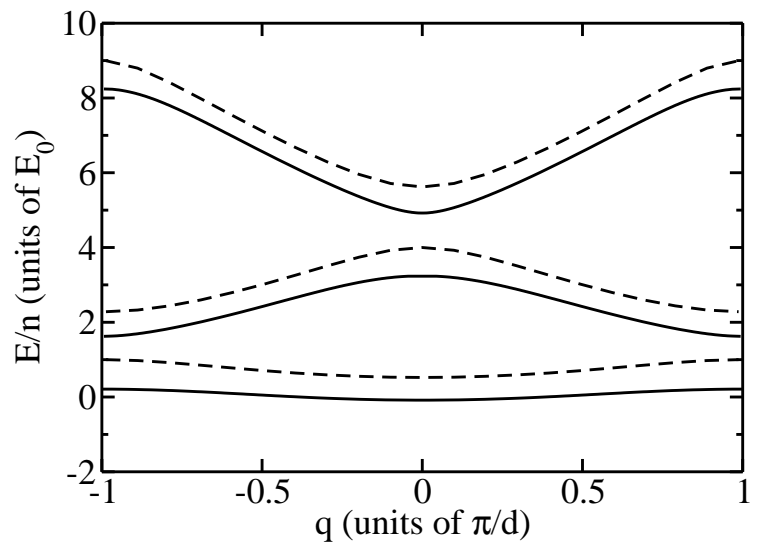

FIG. 4: Energy per particle as a function of the quasimomentum wave number for the first three bands of a weakly attractive condensate $\left(g n=-E_{0}\right)$ in a repulsive lattice $\left(V_{0}=E_{0}\right)$. The noninteracting linear band structure is given by the dashed curves.

most identical to that of the weakly repulsive condensate, Fig. 1 This is to be expected since a weak interaction should only cause small perturbations to a noninteracting system.

In Fig. [5 the band structure for a large attractive interaction, $g n=-10 E_{0}$, in a repulsive potential, $V_{0}=E_{0}$, is illustrated. The energy band for this system is vastly different from the strongly repulsive case. The swallowtails in the bands are now on the upper band at the band gaps as opposed to the lower bands at the band gaps as they were for the repulsive case in Fig. 2. The first energy band never has a swallowtail. This is because the swallowtail must be on the lower portion of the band and below the center of the band there is no quadratic energy dependence for the swallowtail to follow, see Fig. 3 Therefore, the lowest energy band only has a swallowtail for a condensate with repulsive interactions.

The second band of a strongly interacting attractive condensate looks quite different than that of a strongly interacting repulsive condensate. For an attractive condensate, after the initial critical value of the interaction strength is reached, a swallowtail in the band starts to form, as in the third band of Fig. [5] As the interaction strength increases, the width of the swallowtail also increases. Eventually, another critical value is reached where the width of the swallowtail in $q$ reaches $\pi$ and runs into the band edge. If the interaction strength is further increased, the width of the swallowtail increases but the quasimomentum that should be less than zero becomes imaginary due to the form of $\alpha$. This represents a nonphysical solution and is contrary to the assumption that the phase is real in Eq. (8). Therefore, part of the band is absent and the band appears as two separate pieces, a loop and a separate line. These are both marked as Band 2 in Fig. [5] The band takes on this appearance since the swallowtail is attempting to extend 


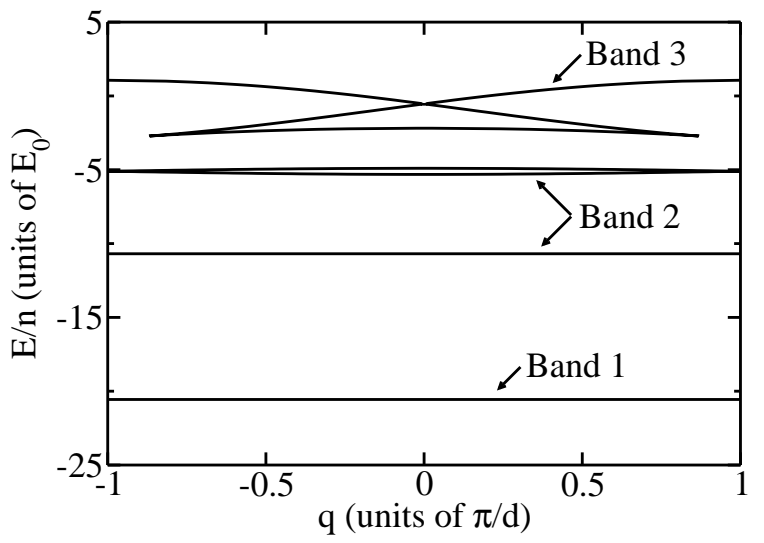

FIG. 5: Energy per particle as a function of the quasimomentum wave number for the first three bands of a strongly attractive condensate $\left(g n=-10 E_{0}\right)$ in a repulsive lattice $\left(V_{0}=E_{0}\right)$. The first band does not have a swallowtail. However, the second and third bands do have swallowtails. The swallowtail in the second band appears as a loop with an unattached curve since an attractive condensate has a maximum width for the swallowtails, which in the case of the second band is a width of $\pi$.

lower than the minimum in the adjacent quadratic energy dependence. In a similar fashion to the first band, the solutions are not physical. The portion of the swallowtail that is before this edge is physical, however, and so a third energy extremum is still present. The third band will exhibit the same behavior when the width of the swallowtail reaches $2 \pi$. In general, the $n^{\text {th }}$ band will appear similar when the swallowtail reaches a width of $(n-1) \pi$ since the swallowtail will then have reached the minimum of the corresponding free particle energy. It should be noted that this phenomenon does not occur for a repulsive interaction since there is no extremum to limit the growth of the swallowtail.

The energy bands are slightly different when the condensate is in an attractive potential. In a manner similar to a repulsive condensate in an attractive potential, for a sufficiently weak interaction strength and large potential strength, the first and second bands can become degenerate.

It should be noted that for a single attractive delta function on the infinite line, there is a critical repulsive interaction above which the impurity can no longer bind the condensate [18]. However, for a periodic system of delta functions, there is no longer a critical value. This can best be understood by considering a condensate of a finite size bound by a single delta function. As the repulsive interaction increases, the condensate spreads out until it reaches the next delta function. This continues indefinitely as the interaction strength increases. There is, therefore, no transition from a bound to unbound state as there was with a single impurity. The symmetry breaking nature of a single impurity allows for the transition that is not present with the symmetric lattice.

\section{DENSITY PROFILES}

As density is the primary experimental observable for BEC's, the change in the density profile as the quasimomentum is varied is important. The case of weak interactions creates similar changes in the density profile and energy band structure independent of the sign of the interaction. This is because the interaction energies are of the same magnitude as the potential. For strong interactions, the density is in general more sharply peaked for an attractive condensate and flatter for a repulsive condensate. However, the general way in which the density changes is qualitatively similar.

The density changes in the first band of the weakly attractive condensate are shown for three different quasimomenta in Fig. [6 The solid curve, dashed curve, and dotted curve represent the density profile for $q=0$, $q=\pi / 2$, and $q=\pi$, respectively. To understand the energy bands in terms of the density profile, the three terms of the energy in Eq. (17) should be discussed. The kinetic, interaction and potential energy per particle are given by,

$$
\begin{aligned}
\frac{E_{k}}{n} & =\frac{1}{n} \int_{0}^{1} \frac{1}{2}\left|\Psi_{x}\right|^{2} \mathrm{~d} x, \\
\frac{E_{i}}{n} & =\frac{1}{n} \int_{0}^{1} \frac{g}{2}|\Psi|^{4} \mathrm{~d} x, \\
\frac{E_{p}}{n} & =\frac{1}{n} \int_{0}^{1} V_{0} \delta(x)|\Psi|^{2} \mathrm{~d} x,
\end{aligned}
$$

respectively. Notice that the density at the origin monotonically decreases as the quasimomentum is increased. Therefore the potential energy will also monotonically decrease due to the delta function at $x=j$, where $j$ is an integer. Since the condensate is attractive, the interaction energy decreases monotonically as the quasimomentum is increased since the density is becoming more peaked. The kinetic energy monotonically increases as the quasimomentum increases since the variations in the density become larger. For a weakly repulsive condensate, the density will also become more strongly peaked as the quasimomentum is increased and will thus have an interaction energy that increases. The larger variations in the density will then increase the kinetic energy as well.

The case of the density variations associated with the first band of a strongly interacting repulsive condensate is shown in Fig. 7 The change in the density is qualitatively similar to that of the weakly attractive case. The solid line represents the density profile for $q=0$. The dot-dash curve represents the density when $q=\pi$ at the bottom of the swallowtail. The dashed curve represents the density when $q=0.46 \pi$ at the end of the swallowtail. The dotted curve represents the density when $q=\pi$ at the top of the swallowtail. The density at the origin again monotonically decreases as the quasimomentum increases and therefore the potential energy also increases monotonically. The interaction energy decreases 

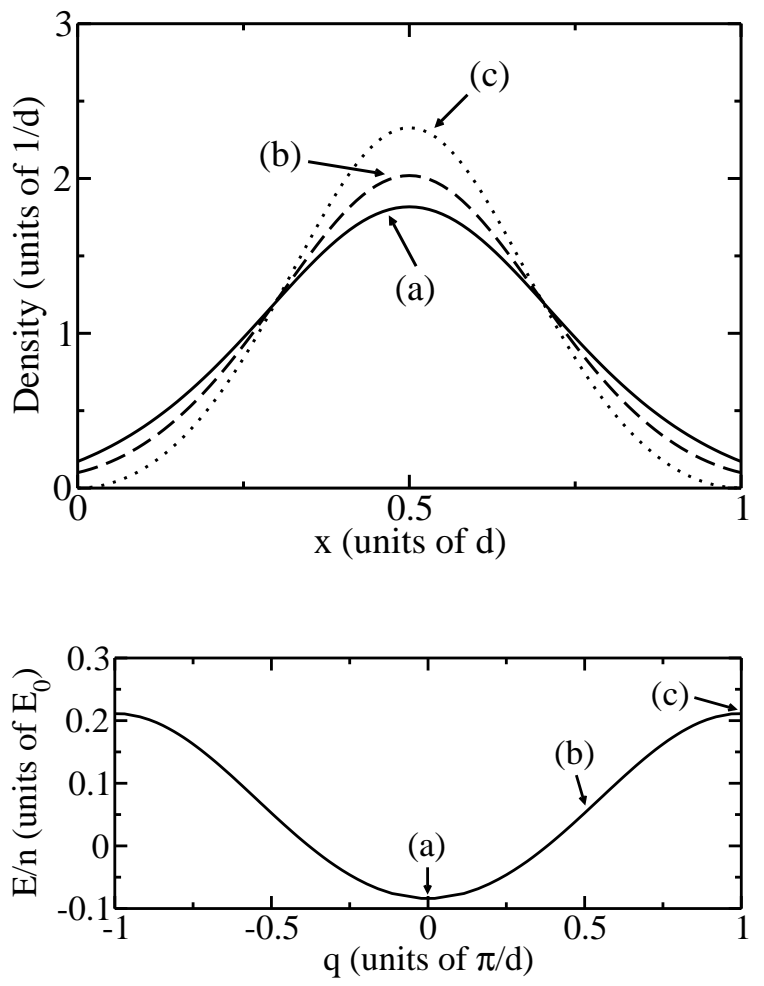

FIG. 6: Changes in the density of a weakly attractive, $g n=$ $-E_{0}$, condensate associated with different positions on the first band. The solid curve represents the density when $q=0$. The dashed curve represents the density when $q=\pi / 2$. The dotted curve represents the density when $q=\pi$. The lower plot shows the corresponding positions on the first energy band.

monotonically as the band is traversed since the density becomes more uniform. The kinetic energy follows the same qualitative path as the total energy. It is therefore the kinetic energy that has the greatest influence on the energy bands.

In the case of strongly attractive interactions, the lowest band consists of well separated bright solitons centered in each lattice site. As the quasimomentum changes from zero to $\pi$, the density changes very little. This is easily noted in the energy spectrum of the lowest band which changes on the order of a tenth of a percent [5]

\section{LIMITING CASES}

It is now shown how the solutions to the NLS connect to the solutions of the linear Schrödinger equation as well as to the discrete nonlinear Schrödinger equation. The linear Schrödinger equation is important since it describes electron motion through crystals. The discrete nonlinear Schrödinger equation describes superfluid systems where the potential strength is much greater than the interaction strength. It is a limit of the Bose-Hubbard model when the Hamiltonian is projected onto a coherent
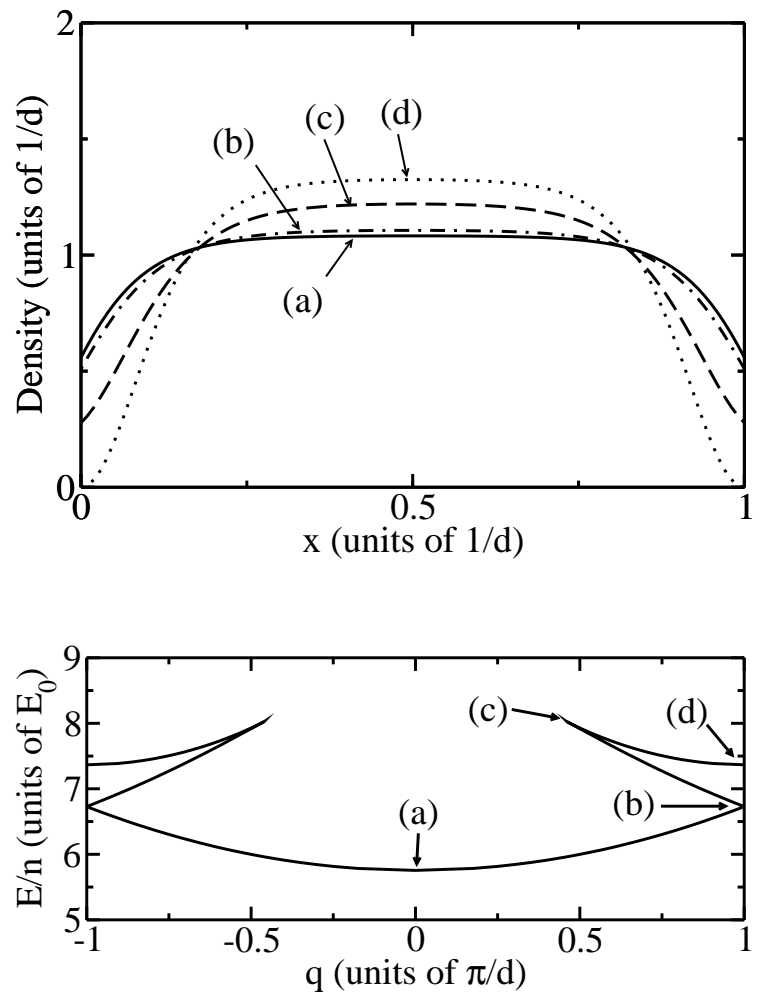

FIG. 7: Changes in the density of a strongly repulsive condensate, $g n=10 E_{0}$, associated with different positions on the first band. The solid curve represents the density when $q=0$. The dot-dashed curve represents the density when $q=\pi$ at the bottom of the swallowtail. The dashed curve represents the density when $q=0.46 \pi$ at the end of the swallowtail. The dotted curve represents the density when $q=\pi$ at the top of the swallowtail. The lower plot shows the corresponding positions on the first energy band.

state [39].

\section{A. Linear Schrödinger Equation Limit}

The energy dispersion relation of the noninteracting regime can be determined from the solutions of the interacting system. In the linear limit, the interaction strength, $g$, and elliptic parameter, $k$, must vanish such that,

$$
\lim _{g, k \rightarrow 0} \frac{k^{2} b^{2}}{g}=A,
$$

where $A$ is the height of the density fluctuations. The elliptic parameter must vanish since in this limit the Jacobi elliptic functions become circular trigonometric functions, which is the appropriate linear form of the density. The linear solutions are then of the form,

$$
\rho(x)=B+A \sin ^{2}(b x+j \pi / 2-b / 2),
$$

where $j$ is a positive integer and $b$ is the horizontal scaling. The offset $j \pi / 2$ can be dropped, since the effect in 
the square of the sine function is to change it into either the square of a sine or a square of a cosine and this merely alters the values of $A$ and $B$. The quasimomentum equation, Eq. (16), can be integrated exactly in the linear limit to find

$$
\cos ^{2}(q / 2)=\frac{1}{1+(1+r) \tan ^{2}(b / 2)},
$$

where $r \equiv A / B$ is the ratio of the density modulation coefficient, $A$, to the constant density offset, $B$. The equation that governs the discontinuity in the derivative of the density at the delta functions, the boundary conditions given by Eq. (10), can be solved for the ratio $r$,

$$
r=-\frac{V_{0}}{b \sin (b / 2) \cos (b / 2)+V_{0} \sin ^{2}(b / 2)} .
$$

The quasimomentum can then be related to the horizontal scaling, $b$, through the relation,

$$
\cos (q)=\cos (b)+\frac{V_{0}}{b} \sin (b) .
$$

This dispersion relationship coincides with that of the linear Schrödinger equation in introductory quantum mechanics texts 56]. At the edges of the Brillouin zone, $q=0$ and $q=\pi$, the heights of the bands and the gaps can be extracted.

\section{B. Discrete Nonlinear Schrödinger Equation Limit}

It is now shown how the solutions to the NLS connect to the solutions of the discrete nonlinear Schrödinger equation (DNLS). The DNLS describes a system where the density is localized in predominantly non-overlapping regions described by the Wannier functions $w_{j}$,

$$
w_{j}\left(x-x_{j}\right) \equiv \frac{1}{\sqrt{N}} \int e^{i q x_{j}} \Psi(x) \mathrm{d} q,
$$

where $x_{j}$ is the position of the $j^{\text {th }}$ lattice site, $N$ is the total number of lattice sites, and the integral is over the first Brillouin zone. Since the regions are non-overlapping, it is required that,

$$
\int w_{j}(x) w_{k}(x) \mathrm{d} x \approx \delta_{j k}
$$

where $w_{j}(x)$ is the Wannier function localized at site $j$ and $\delta_{j k}$ is the Kronecker delta function [3]. This is also known as the tight-binding approximation [57]. In addition, it is assumed that particles can only hop to a nearest neighbor site, i.e. particles in the $j^{\text {th }}$ site can only move to the $(j \pm 1)^{t h}$ site. Therefore, all integrals of the form,

$$
\int\left(\partial_{x} w_{j}^{*}\right)\left(\partial_{x} w_{k}\right) \mathrm{d} x
$$

vanish unless $k \in\{j, j \pm 1\}$. One possible way to create a system like this is to make the strength of the potential sufficiently strong in comparision to the interaction strength of the condensate, $V_{0} / g n \gg 1$.

The DNLS may be extracted from the NLS by using a single band approximation and writing the wavefunction as a linear combination of site-localized Wannier functions,

$$
\Psi(x, t)=\sum_{j} \psi_{j}(t) w_{j}(x),
$$

where $\psi_{j}(t)$ is the probability amplitude to find a particle at site $j$ and $w_{j}(x)$ is the density profile that is localized on site $j$. When Eq. (30) is substituted into Eq. (2), and the spatial degrees of freedom are integrated over, the evolution of the system becomes governed by,

$$
i \partial_{t} \psi_{j}=\epsilon_{j} \psi_{j}+J_{j}\left(\psi_{j+1}+\psi_{j-1}\right)+U_{j}\left|\psi_{j}\right|^{2} \psi_{j},
$$

where $\epsilon_{j}$ is the on-site energy of the $j^{\text {th }}$ site, $J$ is the hopping strength, and $U$ is the interaction strength, with each defined by,

$$
\begin{aligned}
\epsilon_{j} & \equiv V_{0}\left|w_{j}(0)\right|^{2}+\frac{1}{2} \int\left|\partial_{x} w_{j}\right|^{2} \mathrm{~d} x, \\
J_{j} & \equiv \frac{1}{2} \int\left(\partial_{x} w_{j}^{*}\right)\left(\partial_{x} w_{j \pm 1}\right) \mathrm{d} x \\
U_{j} & \equiv g \int\left|w_{j}\right|^{4} \mathrm{~d} x .
\end{aligned}
$$

The stationary states of the DNLS are given by plane waves, $\psi_{j}=\psi_{0} e^{i(p j-\mu t)}$, where $p$ is the momentum and the eigenvalue, $\mu$, is given by,

$$
\mu=U\left|\psi_{0}\right|^{2}+\epsilon+2 J \cos (p) .
$$

The subscript $j$ has been dropped since all Wannier functions have the same profile, only translationally shifted to the appropriate site. The energy of the condensate for each site is given by,

$$
E_{\text {site }}=\frac{1}{2} U\left|\psi_{0}\right|^{4}+\left|\psi_{0}\right|^{2} \epsilon+2\left|\psi_{0}\right|^{2} J \cos (p) .
$$

If the Wannier functions are normalized to unity, then $\psi_{0}=\sqrt{n}$ and the equation for the energy per particle becomes,

$$
\frac{E_{\text {site }}}{n}=\frac{1}{2} U N+\epsilon+2 J \cos (p) .
$$

The Wannier functions for the Kronig-Penney potential can be extracted from Eq. (11) by taking the limit when the potential strength approaches infinity.

It should be noted that a large attractive interaction in a repulsive potential will have a similar effect as a large repulsive potential and hence the DNLS can be used to describe the system. In this case, the large attractive interaction causes the density of each site to approach a hyperbolic secant squared profile, i.e., a bright soliton. 
Hence, $k \rightarrow 1$ and $B \rightarrow-b^{2} / g$. The normalization of the density fixes $b=g n / 2$ and the quasimomentum becomes zero. This density profile is the equivalent of the Wannier functions discussed earlier. The on-site energy is calculated to be,

$$
\epsilon=-g n V_{0} \exp [g n / 2]+\frac{g^{2} n^{2}}{24} .
$$

The interaction term becomes,

$$
U=-\frac{g^{2} n^{2}}{6} .
$$

With the assumption that $|g|$ is large, the hopping term is given by,

$$
J=\frac{g^{2} n^{2}}{8}(4+g n) \exp [g n / 2] .
$$

The DNLS energy, Eq. (36), becomes consistent with the NLS energy, Eq. (17), to within three tenths of a percent for the first band of the strongly attractive case, $g n=-10 E_{0}$ and $V_{0}=E_{0}$. When the on-site energy, interaction energy, and hopping strength are all assumed to remain constant, the maximum error in energy remains within three tenths of a percent across the entire band. The energy is slightly underestimated at zero quasimomentum and is slightly overestimated at $\pi$ quasimomentum. In this instance, knowing the density profile at one value of the quasimomentum allows for extension to the full spectrum of quasimomentum. When the interaction energy becomes strong enough that only terms to highest order of $g$ need to be considered, the total energy becomes $-g^{2} n^{2} / 24$ and the variation across the band becomes $-g^{3} n^{3} / 2 \exp [g n / 2]$, which is essentially flat.

\section{STABILITY}

We proceed to study the stability of the Bloch states and to determine the stable regions of the bands are determined. In addition to stable solutions, solutions that have instability times much longer than experimental time scales can be observed in experiments. Recent studies of the stability of condensates in a periodic potential have focused on linear energetic and dynamic stability, also called Landau stability [6, 22, 43, 47, 58]. In contrast, we consider the full response of the condensate to stochastic perturbations. In order to numerically simulate the NLS with a periodic potential, a ring geometry is used with a quantized phase. To ensure that the phase quantization does not effect the stability properties, enough lattice sites were used to allow for many rotations of the phase such that $N q=2 \pi j$, where $q$ is the quasimomentum, $N$ is the number of sites nad $j$ is an interger. The outcome of the stability analysis is independent of the number of sites for sufficiently large number of sites. In most cases, $j=4$ was found to be adequate to extract the correct stability properties.
The delta functions are simulated by single point distortions in the potential grid. They are also implemented by using boxes of different widths with their area normalized to create the appropriate potential strength. The size of the boxes did not influence the stability properties until the width became approximately $10 \%$ of the size of the healing length, $\xi \equiv \hbar / \sqrt{2 g n}$. The NLS is evolved using a variable step fourth-order Runge-Kutta algorithm in time and a filtered pseudo-spectral method in space. The noise introduced into the simulations comes from the round off error associated with numerical simulations. To ensure that the form of the noise from round off error did not affect the stability properties of the system, initial stochastic white noise of various levels was introduced into the Fourier spectrum. For levels significantly greater than the round off noise, the stability times approached those from the round off noise. Introduction of white noise at the level in the eighth significant digit produced the same instability times as the round off noise, which effects the sixteenth significant digit. All simulations were performed over time scales longer than experimental lifetimes of the BEC, which are on the order of seconds.

The time at which the onset of instability occurs is determined by the effective variance in the Fourier spectrum,

$$
\sigma(t) \equiv \sqrt{\frac{\sum(f(p, t)-f(p, 0))^{2}}{2 \sum(f(p, 0))^{2}}},
$$

where $f(p, t)$ is the Fourier component of the wavefunction at momentum $p$ and time $t$ and the sum is over the momentum grid. This quantity determines how different the Fourier spectrum is compared to the original stationary state. It vanishes when the two spectrums are identical and approaches unity when there are no Fourier components in common. When $\sigma(t)$ reaches 0.5 , i.e. $50 \%$ of the Fourier spectrum is different than the original, the system is considered to have become unstable.

Unless otherwise noted, for the stability analysis the lattice spacing is given by $d=1 \mu \mathrm{m}$, the length scale with which current optical lattices are created. In addition, all instability time scales will be given for ${ }^{87} \mathrm{Rb}$.

\section{A. Attractive Atomic Interactions}

With an attractive interaction of $g n=-E_{0}$ in a repulsive potential of $V_{0}=E_{0}$ (see Fig. (4), the lowest energy solution, zero quasimomentum in the lowest band, has a lifetime greater than experimental time scales. However, when even a slight harmonic perturbation to the potential is added to the initial time step, for instance a harmonic frequency of $120 \mathrm{~Hz}$ which is approximately the experimental trapping frequency [59] in the longitudinal dimension, the condensate becomes unstable on time scales on the order of $1.5 \mathrm{~ms}$. This is short compared to the lifetime of a BEC [59], but is still observable. 
When the quasimomentum of the first band is increased, the stability of the system becomes dependent on the effective mass. The effective mass, $m^{*}$, is defined by [60],

$$
m^{*} \equiv \frac{1}{\partial^{2} E / \partial q^{2}}
$$

The effective mass is the mass that the particle appears to have if the potential was not being considered [1]. The sign of the effective mass can be transferred to the interaction strength, changing an attractive interaction to a repulsive interaction. Therefore, when the quasimomentum increases and the energy band becomes concave down, $m^{*}<0$, the system enters a regime of stability. The system remains stable even in the presence of the harmonic perturbation.

For zero quasimomentum in the second band, the system immediately possesses periodic variations in the phase and density. There is an additional underlying instability that occurs on the order of $5 \mathrm{~ms}$ that destroys the periodicity of the system. It would be expected that this part of the band be stable since there is a negative effective mass but the oscillations due to the two bright solitons per lattice site force the system to be unstable. The oscillations, although periodic in time, create a larger underlying instability to grow.

The stability properties of a strongly attractive condensate are similar to those of a weakly attractive condensate. The stability of the first band is determined by the effective mass while higher bands always go unstable. Therefore, for an attractive condensate, the system of Bloch waves is stable only if there is one soliton per lattice site and the effective mass is negative.

\section{B. Repulsive Atomic Interactions}

Similar to an attractive condensate, a repulsive condensate only has stable regions on the first band. For a weakly interacting repulsive condensate, $g n=E_{0}$ and $V_{0}=E_{0}$, the effective mass in the first band is positive between $q=0$ and $q=\pi / 2$. The effective mass then becomes negative for larger quasimomentum since the energy becomes concave down and hence the system becomes unstable. For a quasimomentum of $q=9 \pi / 16$ the instability time is $10 \mathrm{~ms}$ and reduces to $2 \mathrm{~ms}$ for $q=\pi$. In this regime, with negative effective mass, the ground state is an envelope soliton that can spread over many lattice sites. These types of states are called gap solitons [7, 33, 37, 61] and only occur in interacting systems. Figure 8 presents the unstable evolution of the weakly repulsive condensate in the first band with a quasimomentum of $q=\pi$ in Fourier space. Notice that the instabilities arise from perturbations around the primary Fourier components of the wavefunction. In Fig. 9 the effective variance, $\sigma$, is plotted as a function of evolution time and the system becomes unstable around $2 \mathrm{~ms}$. The second band becomes unstable when $q=0$ in $0.500 \mathrm{~ms}$

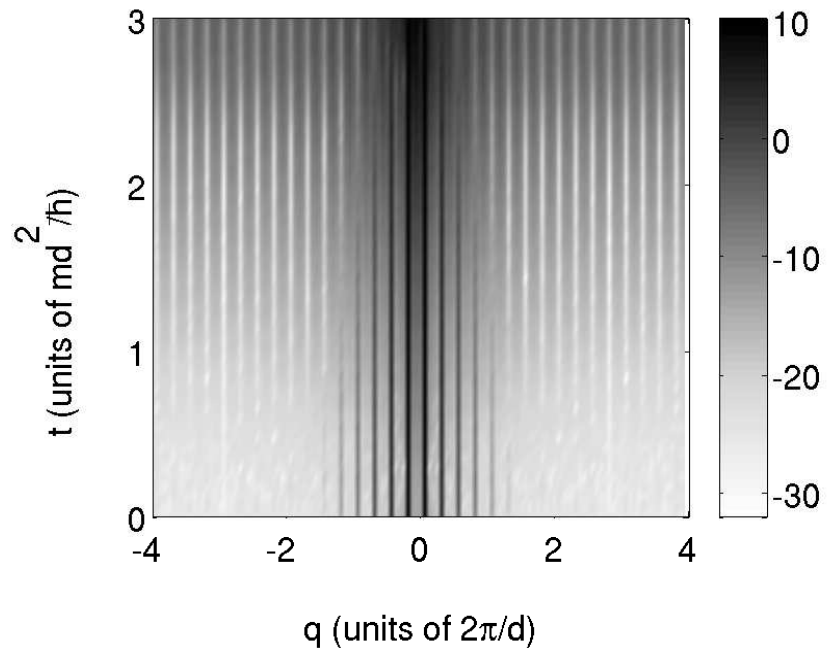

FIG. 8: Logarithm of the Fourier spectrum during the time evolution. Time is in units of $\hbar / E_{0}$ and distance in units of the lattice spacing.

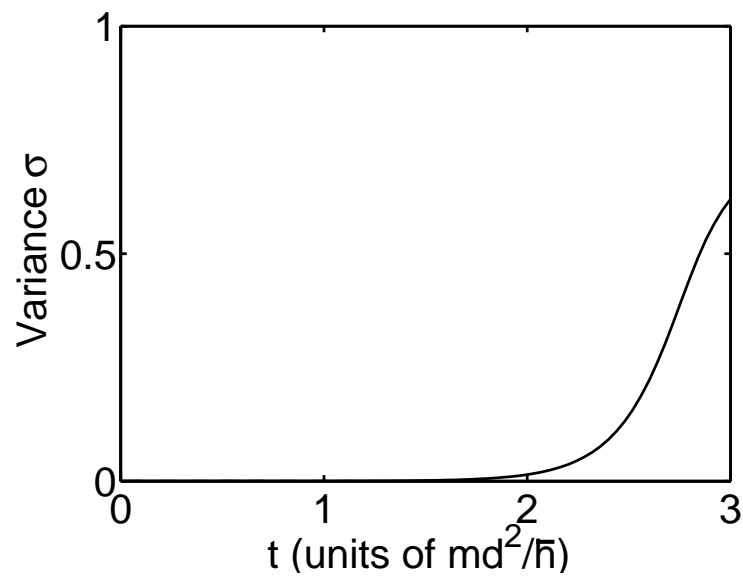

FIG. 9: The time evolution of the effective variance of the momentum density, $\sigma$. Time is in units of $\hbar / E_{0}$.

and in $6 \mathrm{~ms}$ for $q=\pi$. Therefore, the system is stable in the first band with positive effective mass, $m^{*}>0$, and unstable elsewhere. This is consistent with the effects that the effective mass has on stability in systems described by the lowest band DNLS. In a work by Fallani et al. [6], the instability time of a condensate in a lattice was measured by using an RF-shield to remove the hottest atoms produced by the heating created in the sample due to instability. The loss rate, given by the inverse of the lifetime, should then be qualitatively similar to the instability time. Our calculations are consistent with these experimentally observed loss rates of a BEC in an optical lattice [6].

Due to the presence of the swallowtails, the strongly interacting system provides different stability regimes. For 


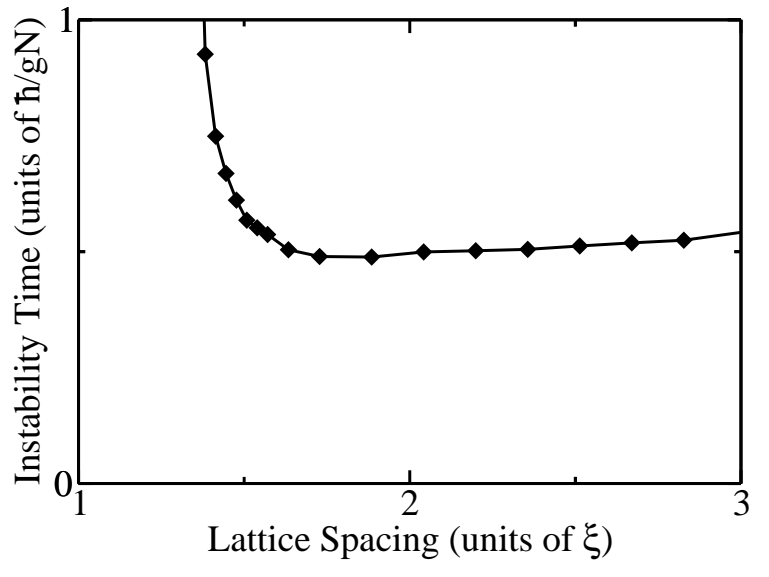

FIG. 10: The time to instability as the lattice spacing is varied. Time is in unit of $\hbar / g n$ and distance in units of the healing length, $\xi=\hbar / \sqrt{2 g n}$.

a repulsive condensate, $g n=10 E_{0}$, in a repulsive lattice, $V_{0}=E_{0}$, the main section of the first band, as well as the lower portion of the swallowtail, have positive effective mass and remain stable. The upper portion of the swallowtail, as discussed in Sec. IIII is an energy maximum and is not expected to remain stable. In fact, the instability time of the upper portion of the swallowtail is approximately $0.2 \mathrm{~ms}$, independent of the actual quasimomentum.

The size of the lattice spacing can influence the time scales for which the system becomes unstable. In Fig.10 the time to instability is presented as a function of the lattice spacing for a repulsive condensate, $g n=E_{a}$, in a repulsive lattice, $V_{0}=E_{a}$, with a quasimomentum of $q=\pi$, where $E_{a} \equiv \hbar^{2} \pi^{2} / 2 m(1 \mu m)^{2}$.

There is a minimum instability time as the lattice spacing varies that occurs when the lattice spacing is approximately twice the healing length, $\xi$. The time to instability is given by half of the interaction strength time, $g n / \hbar$. When the lattice spacing is much larger than the healing length, the density becomes extremely flat except at the delta functions, where dark solitons form. Since the lattice spacing is large, the dark solitons are far apart and are effectively noninteracting pinned solitons. Dark solitons are known to be robustly stable [44, 55] and, therefore, for a lattice spacing much larger than the healing length the system should become stable. For lattice spacing smaller than twice the healing length, the condensate has difficulty distinguishing between the separate delta functions and sees closer to a constant potential. In this regime, the kinetic energy becomes much greater than the interaction energy and potential energy since variations in the density occur on the length scale of $d / 2$, which is less than the healing length. The system then becomes effectively free and noninteracting and, therefore, approaches stability.

\section{DISCUSSION AND CONCLUSIONS}

The full set of stationary states of a Bose-Einstein condensate in a Kronig-Penney lattice potential, with period commensurate with the lattice, have been presented analytically in the form of Bloch waves for both repulsive and attractive interactions. The quasimomentum energy bands were found to exhibit a cusp at the critical interaction strength $g n=2 V_{0}$, where $g$ is the interatomic interaction strength, $n$ is the linear density, and $V_{0}$ is the lattice potential strength. For larger interaction strengths, swallowtails form in the bands. These swallowtails have the same qualitative form as for a sinusoidal potentail and exhibit the same stability properties.

Both attractive and repulsive condensates were found to be dynamically stable only in the first band and when the effective interaction, $\operatorname{sgn}\left(m^{*}\right) g$, was positive. Also, even in the first band, the upper edges of swallowtails were always unstable. It should be noted that the effective mass does not influence the stability of the higher bands since they are always unstable. Therefore, for an attractive condensate, the only stable Bloch states exist in the first band between the quasimomentum where $m^{*}$ becomes negative and $q=\pi$. A repulsive condensate is only stable in the first band from $q=0$ to the quasimomentum where $m^{*}$ becomes negative. Higher bands will always be unstable, for both attractive and repulsive condensates. When solutions became unstable, our numerically studies consistently observed that the instabilities originated around the primary Fourier components of the wavefunction. This is in agreement with the formal proof of the instability of constant phase Bloch-type solutions by Bronski et al. [44]. The instability time was found to be a function of the lattice constant. If the delta functions are spaced either smaller than or much larger than the healing length of the condensate, the solutions had instability times longer than lifetime of the BEC. Thus experiments could access formally unstable sections of the energy bands, and, by controlling the ratio of the healing length to the lattice constant, directly observe the dynamics of instability. The results of our stability analysis are consistent with the experimental work performed by Fallani et al. [6], in which the loss rate, the inverse of the lifetime, was determined by removing the hottest atoms with an RF-shield.

An interesting phenomenon to note is that for a repulsive condensate when a swallowtail is present, the entire energy band is concave up and, hence, the effective mass is always positive. This is in contrast to a weakly repulsive condensate, when the concavity of the energy band changes, creating a region of negative effective mass. Therefore, there is a maximum interaction strength for which gap solitons [3, 7, 33, 37, 61] can be formed since they require a negative effective mass, which does not occur when a swallowtail is present. The maximum interaction energy is given by $g n=2 V_{0}$, the strength at which swallowtails appear.

Stationary solutions to the NLS with a Kronig-Penney 
potential need not take the form of Bloch states. Solutions with a period which is an integer multiple of the lattice period have been shown to exist for the sinusoidal potential [58] and are expected also to be present for the Kronig-Penney potential. Envelope solutions, such as gap solitons, also play an important role in other systems modelled by the NLS and have been observed in BEC's 7]. The analytic methods which we have described here are equally applicable to these solution types and will form the subject of future study 62 .

\section{Acknowledgments}

We acknowledge helpful discussions with John Cooper, Chris Pethick, Ana Maria Rey, Augusto Smerzi, and Eugene Zaremba. Support is acknowledged for B.T.S. from the National Science Foundation and for L.D.C. and M.J.H. from the U.S. Department of Energy, Office of Basic Energy Sciences via the Chemical Sciences, Geosciences and Biosciences Division.
[1] S. L. Altmann, Band Theory of Metals: The Elements (Pergamon PRess, Oxford, 1970).

[2] R. A. Usmanov and L. B. Ioffe, Phys. Rev. B 69, 214513 (2004).

[3] D. N. Christodoulides, F. Lederer, and Y. Silberberg, Nature 424, 817 (2003).

[4] M. Greiner, I. Bloch, O. Mandel, T. W. Hansch, and T. Esslinger, Appl. Phys. B 73, 769 (2001).

[5] L. Fallani, F. S. Cataliotti, J. Catani, C. Fort, M. Modugno, M. Zawada, and M. Inguscio, Phys. Rev. Lett. 91, 240405 (2003).

[6] L. Fallani, L. De Sarlo, J. E. Lye, M. Modugno, R. Saers, C. Fort, and M. Inguscio, Phys. Rev. Lett. 93, 140406 (2004).

[7] B. Eiermann, T. Anker, M. Albiez, M. Taglieber, P. Treutlein, K. P. Marzlin, and M. K. Oberthaler, Phys. Rev. Lett. 92, 230401 (2004).

[8] B. Eiermann, P. Treutlein, T. Anker, M. Albiez, M. Taglieber, K.-P. Marzlin, and M. K. Oberthaler, Phys. Rev. Lett. 91, 060402 (2003).

[9] T. Esslinger and K. Molmer, Phys. Rev. Lett. 90, 160406 (2003).

[10] M. Greiner, O. Mandel, T. Esslinger, T. W. Hansch, and I. Bloch, Nature 415, 39 (2002).

[11] B. P. Anderson and M. A. Kasevich, Science 282, 1686 (1998).

[12] E. W. Hagley, L. Deng, M. Kozuma, J. Wen, K. Helmerson, S. L. Rolston, and W. D. Phillips, Science 283, 1706 (1999).

[13] Y. B. Ovchinnikov, J. H. Müller, M. R. Doery, E. J. D. Vredenbregt, K. Helmerson, S. L. Rolston, and W. D. Phillips, Phys. Rev. Lett. 83, 284 (1999).

[14] J. L. Roberts, N. R. Claussen, J. P. Burke, Jr., C. H. Greene, E. A. Cornell, and C. E. Wieman, Phys. Rev. Lett. 81, 5109 (1998).

[15] A. Inouye, M. R. Andrews, J. Stenger, H.-J. Miesner, D. M. Stamper-Kurn, and W. Ketterle, Nature 392, 151 (1998).

[16] E. P. Gross, Nuovo Cimento 20, 454 (1961).

[17] L. P. Pitaevskii, Zh. Eksp. Teor. Fiz. 40, 646 (1961).

[18] B. T. Seaman, L. D. Carr, and M. J. Holland, cond-mat/0410345 (2004).

[19] G. E. Astrakharchik and L. P. Pitaevskii, Phys. Rev. A 70, 013608 (2004).

[20] A. Radouani, Phys. Rev. A 70, 013602 (2004).

[21] M. M. Bogdan, A. S. Kovalev, and I. V. Gerasimchuk, Low Temp. Phys. 23, 145 (1997).

[22] M. Machholm, C. J. Pethick, and H. Smith, Phys. Rev.
A 67, $053613(2003)$.

[23] B. Wu, R. B. Diener, and Q. Niu, Phys. Rev. A 65, 025601 (2002).

[24] E. J. Mueller, Phys. Rev. A 66, 063603 (2002).

[25] A. M. Rey, K. Burnett, R. Roth, M. Edwards, C. J. Williams, and C. W. Clark, J. Phys. B 36, 825 (2003).

[26] M. H. Anderson, J. R. Ensher, M. R. Matthews, C. E. Wieman, and E. A. Cornell, Science 269, 198 (1995).

[27] K. B. Davis, M.-O. Mewes, M. R. Andrews, N. J. van Druten, D. S. Durfee, D. M. Kurn, and W. Ketterle, Phys. Rev. Lett. 75, 3969 (1995).

[28] P. Meystre and M. Sargent III, Elements of Quantum Optics, 3rd ed. (Springer, Berlin, 1999).

[29] J. I. Cirac and P. Zoller, Phys. Today 57, 38 (2004).

[30] M. Girardeau, J. Math. Phys. 1, 516 (1960).

[31] B. Paredes, A. Widera, V. Murg, O. Mandel, S. Folling, Cirac, G. V. Shlyapnikov, T. W. Hänsch, and I. Bloch, Nature 429, 277 (2004).

[32] T. Kinoshita, T. Wenger, and D. S. Weiss, Science 305, 1125 (2004).

[33] G. Lenz, P. Meystre, and E. M. Wright, Phys. Rev. A 50, 1681 (1994).

[34] D. Diakonov, L. M. Jensen, C. J. Pethick, and H. Smith, Phys. Rev. A 66, 013604 (2002).

[35] P. Massignan and M. Modugno, Phys. Rev. A 67, 023614 (2003).

[36] E. A. Ostrovskaya and Y. S. Kivshar, Phys. Rev. Lett. 92, 180405 (2004).

[37] P. J. Y. Louis, E. A. Ostrovskaya, C. M. Savage, and Y. S. Kivshar, Phys. Rev. A 67, 013602 (2003).

[38] B. Wu and Q. Niu, Phys. Rev. A 64, 061603(R) (2001).

[39] A. M. Rey, Ph.D. thesis, University of Maryland at College Park, 2004.

[40] B. Deconinck, B. A. Frigyik, and J. N. Kutz, J. Nonlinear Sci. 12, 169 (2002).

[41] E. Taylor and E. Zaremba, Phys. Rev. A 68, 053611 (2003).

[42] B. Wu and Q. Niu, Phys. Rev. A 61, 023402 (2001).

[43] J. C. Bronski, L. D. Carr, B. Deconinck, and J. N. Kutz, Phys. Rev. Lett. 86, 1402 (2001).

[44] J. C. Bronski, L. D. Carr, B. Deconinck, J. N. Kutz, and K. Promislow, Phys. Rev. E 63, 036612 (2001).

[45] M. A. Porter, P. G. Kevrekidis, and B. A. Malomed, Physica D 196, 106 (2004).

[46] W.-D. Li and A. Smerzi, Phys. Rev. E 70, 016605 (2004).

[47] D. Taras-Semchuk and J. M. F. Gunn, Phys. Rev. B 60, 13139 (1999).

[48] S. A. Alexander and R. L. Coldwell, Int. J. of Qu. Chem. 
86, 325 (2002).

[49] S. Theodorakis and E. Leontidis, J. Phys. A 30, 4835 (1997).

[50] Y. B. Gaididei, P. L. Christiansen, K. O. Rasmussen, and M. Johansson, Phys. Rev. B 55, R13365 (1997).

[51] L. D. Carr, M. A. Leung, and W. P. Reinhardt, J. Phys. B 33, 3983 (2000).

[52] M. Olshanii, Phys. Rev. Lett. 81, 938 (1998).

[53] Handbook of Mathematical Functions, edited by M. Abramowitz and I. A. Stegun (National Bureau of Standards, Washington, D.C., 1964).

[54] L. M. Milne-Thomson, Jacobian Elliptic Function Tables (Dover Publications Inc., New York, 1950).

[55] Y. S. Kivshar and B. Luther-Davies, Physics Reports 298, 81 (1998).
[56] R. L. Liboff, Introductory Quantum Mechanics, fourth ed. (Addison Wesley, San Francisco, 2003).

[57] A. Smerzi and A. Trombettoni, Phys. Rev. A 68, 023613 (2003).

[58] M. Machholm, A. Nicolin, C. J. Pethick, and H. Smith, Phys. Rev. A 69, 043604 (2004).

[59] H. Moritz, T. Stöferle, M. Köhl, and T. Esslinger, Phys. Rev. Lett. 91, 250402 (2003).

[60] Note one can also define an effective mass associated with the chemical potential bands as in Li and Smerzi [46].

[61] K. M. Hilligsoe, M. K. Oberthaler, and K.-P. Marzlin, Phys. Rev. A 66, 063605 (2002).

[62] B. T. Seaman, L. D. Carr, and M. J. Holland, work in progress (2005). 